\title{
Modeling the effect of subsurface interface defects on contact stiffness for ultrasonic atomic force microscopy
}

A. F. Sarioglu, A. Atalar, and F. L. Degertekin

Citation: Appl. Phys. Lett. 84, 5368 (2004); doi: 10.1063/1.1764941

View online: http://dx.doi.org/10.1063/1.1764941

View Table of Contents: http://aip.scitation.org/toc/apl/84/26

Published by the American Institute of Physics

\section{Articles you may be interested in}

Contact stiffness of finite size subsurface defects for atomic force microscopy: Three-dimensional finite element modeling and experimental verification

Journal of Applied Physics 103, 114910 (2008); 10.1063/1.2936881

Relationship between Q-factor and sample damping for contact resonance atomic force microscope measurement of viscoelastic properties

Journal of Applied Physics 109, 113528 (2011); 10.1063/1.3592966

Contact stiffness of layered materials for ultrasonic atomic force microscopy

Journal of Applied Physics 87, 7491 (2000); 10.1063/1.373014

Thin film characterization by atomic force microscopy at ultrasonic frequencies

Applied Physics Letters 76, 1950 (2000); 10.1063/1.126222

Ultrasonic force microscopy for nanometer resolution subsurface imaging

Applied Physics Letters 64, 178 (1998); 10.1063/1.111524

Vibrations of free and surface-coupled atomic force microscope cantilevers: Theory and experiment

Review of Scientific Instruments 67, 3281 (1998); 10.1063/1.1147409

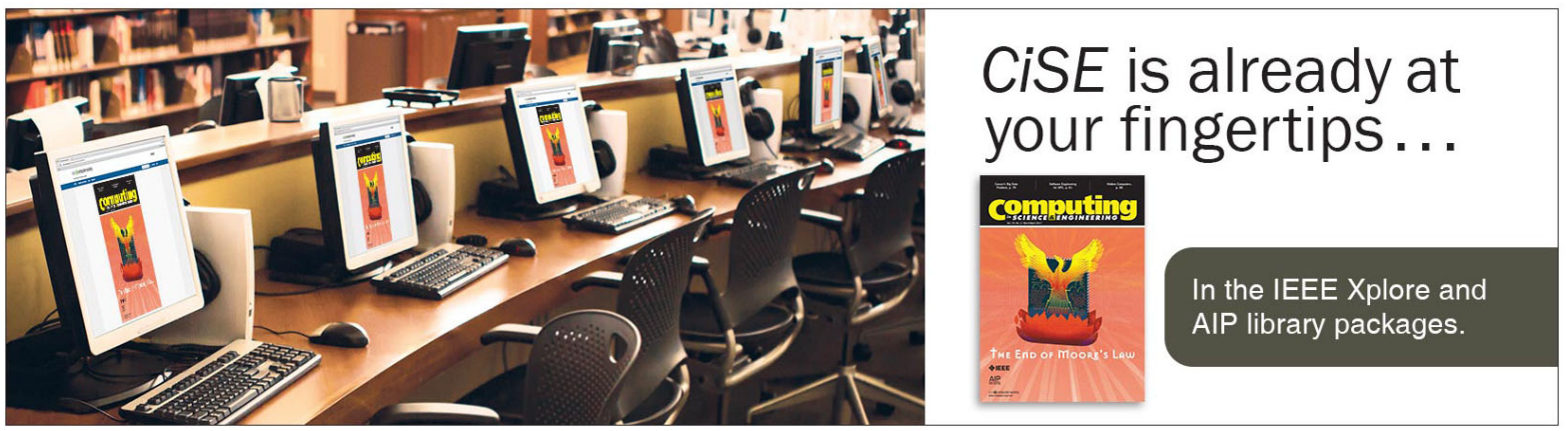




\title{
Modeling the effect of subsurface interface defects on contact stiffness for ultrasonic atomic force microscopy
}

\author{
A. F. Sarioglua) and A. Atalar \\ Department of Electrical and Electronics Engineering, Bilkent University, Ankara, 06800, Turkey \\ F. L. Degertekin \\ G. W. Woodruff School of Mechanical Engineering, Georgia Institute of Technology, Atlanta, \\ Georgia 30332
}

(Received 19 January 2004; accepted 28 April 2004; published online 17 June 2004)

\begin{abstract}
We present a model predicting the effects of mechanical defects at layer interfaces on the contact stiffness measured by ultrasonic atomic force microscopy (AFM). Defects at subsurface interfaces result in changes at the local contact stiffness between the AFM tip and the sample. Surface impedance method is employed to model the imperfections and an iterative algorithm is used to calculate the AFM tip-surface contact stiffness. The sensitivity of AFM to voids or delaminations and disbonds is investigated for film-substrate combinations commonly used in microelectronic structures, and optimum defect depth for maximum sensitivity is defined. The effect of contact force and the tip properties on the defect sensitivity are considered. The results indicate that the ultrasonic AFM should be suitable for subsurface detection and its defect sensitivity can be enhanced by adjusting the applied force as well as by judicious choice of the AFM tip material and geometry. (C) 2004 American Institute of Physics. [DOI: 10.1063/1.1764941]
\end{abstract}

Mapping of elastic properties at the nanoscale has become a significant application of atomic force microscopy (AFM) in the recent years. Several methods based on low frequency indentation, ${ }^{1}$ tapping mode, ${ }^{2}$ and AFM at ultrasonic frequencies ${ }^{3,4}$ have been proposed and used to image the surface stiffness of various samples in air and in liquids. ${ }^{5}$ All of these methods acquire elasticity information through the AFM tip - sample surface contact stiffness, which is usually modeled as a simple spring and is a function of the sample elasticity as well as the geometry and the material of the AFM tip.

Although the response of the AFM to elastic properties is mostly determined by a volume close to the sample surface, it can be shown that the stress fields generated by the AFM tip also provide a penetration depth, ${ }^{6}$ albeit limited, for subsurface defect imaging. For example, this capability of ultrasonic AFM has enabled researchers to visualize subsurface dislocations in highly ordered pyrolytic graphite (HOPG), and debonding of cracked nanocomposite films on polymer substrates in situ while the substrate material is stressed. ${ }^{7,8}$ These initial results show the potential of ultrasonic AFM for significant problems such as nondestructive characterization of new materials for microelectronics, and detection and imaging of defects in electrical interconnects and other thin film devices with nanoscale lateral resolution. ${ }^{9-11}$ In order to perform quantitative AFM imaging of layered materials and defects at subsurface interfaces, a surface impedance based approach combined with an iterative algorithm was recently developed to model the contact stiffness of thin film/substrate structures for ultrasonic AFM. ${ }^{6}$ The efficacy of the method in predicting flexural resonance spectra of AFM cantilevers has been verified by experiments. ${ }^{12}$

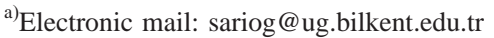

In this letter, we incorporate interface defects into the surface impedance based contact stiffness calculations to predict the response of the AFM to buried interface defects such as disbonds, delaminations and voids in complex, multilayered structures. We calculate the sensitivity of ultrasonic AFM to subsurface defects typically found in electrical interconnects and integrated circuits as examples. Optimization of AFM parameters, such as tip radius, contact force and tip material for defect detection, and comparison of sensitivity to particular defects are also discussed.

Unlike the Hertz solution of the contact between two half spaces, analytical calculation of contact stiffness of a multilayered sample is rather involved, and finite element methods used for this problem suffer from convergence problems requiring excessive computational power. To overcome these difficulties we use the surface impedance based contact stiffness calculation algorithm. ${ }^{6}$ This algorithm makes use of a variational formula [Eq. (1)] to determine the mechanical radiation impedance, $Z(\omega)$, of the sample where $\omega$ is the angular frequency. Assuming that the AFM tip applies normal stress over the contact area, i.e., shear stresses at the surface vanish, and the contact acts as an ultrasonic radiator with a circularly symmetric stress distribution, one can write the expression for $Z(\omega)$ in Fourier domain as

$$
Z(\omega)=-\int_{0}^{\infty}\left|T_{z z}\left(k_{r}\right)\right|^{2} k_{r} d k_{r} / \int_{0}^{\infty} T_{z z}^{*}\left(k_{r}\right) V_{z}\left(k_{r}\right) k_{r} d k_{r},
$$

where $T_{z z}$ is the stress component normal to the sample surface, $V_{z}$ is the particle velocity, and $k_{r}$ is the radial wave number of the particular plane wave component radiated from the contact. In order to use Eq. (1) for contact stiffness, the relation between the stress and particle velocity fields at the surface of the multilayered substrate needs to be known. The relation can be obtained by defining the surface impedance tensor $\mathbf{G}$ at the sample surface by 


$$
\mathbf{T}=\mathbf{G V} \Rightarrow\left[\begin{array}{l}
T_{x z} \\
T_{y z} \\
T_{z z}
\end{array}\right]=\left[\begin{array}{lll}
G_{11} & G_{12} & G_{13} \\
G_{21} & G_{22} & G_{23} \\
G_{31} & G_{32} & G_{33}
\end{array}\right]\left[\begin{array}{c}
V_{x} \\
V_{y} \\
V_{z}
\end{array}\right],
$$

where $T_{x z}, T_{y z}$, and $T_{z z}$ are stress fields acting on the $x-y$ plane and $V_{x}, V_{y}$, and $V_{z}$ are the particle velocities in the respective directions. As $T_{z z}$ is the only nonzero stress field imposed by the tip, the acoustic power radiated into the sample will be determined by the $z$ component of the particle velocity vector, $V_{z}\left(k_{r}\right)=G_{33}^{-1}\left(k_{r}\right) T_{z z}\left(k_{r}\right)$. Assuming a uniform stress distribution over contact area of AFM tip on the sample, i.e., $T_{z z}\left(k_{r}\right)=2 \pi a J_{1}\left(a k_{r}\right) / k_{r}$, we arrive at

$$
Z(\omega)=\frac{-\pi a^{2}}{2 \pi a^{2} \int_{0}^{\infty}\left[\left.\left[\frac{J_{1}\left(a k_{r}\right)}{k_{r}}\right]\right|^{2} G_{33}^{-1}\left(k_{r}\right) k_{r} d k_{r}\right.},
$$

where $J_{1}($.$) is the first-order Bessel function of the first kind$ and $G_{33}^{-1}$ is the element with index 3,3 in the inverse of surface impedance matrix at the surface.

For time harmonic wave motion, we can relate it to the surface stiffness, $k_{s}$, which is force/average displacement using the relation $k_{s}=j \omega A Z$, where $A=\pi a^{2}$ is the contact area, $a$ is the contact radius. Comparisons with analytical and finite element results show that for AFM applications, where the contact radius is small as compared to wavelength of acoustic waves, this quasistatic lumped element approximation is accurate for frequencies from dc up to $\mathrm{GHz}$ range. ${ }^{6}$

For a multilayered sample, this procedure allows one to include different types of interface defects such as voids, delaminations, and disbonds at the desired subsurface interface by imposing particular boundary conditions on the surface impedance tensors. ${ }^{13}$ Electromigration induced voids, and delaminations between the low- $k$ dielectric-metal interfaces in electrical interconnects can be considered as important practical examples. ${ }^{10,11} \mathrm{~A}$ delamination or void at an interface means that none of the stress components can be sustained, whereas nonzero particle displacements are possible. According to the definition in Eq. (2), the suitable initial condition on the surface impedance tensor at the interface becomes

$$
T=0, V \neq 0 \Rightarrow G=0 .
$$

In the case of a disbond or a so-called "kissing bond," the shear stress vanishes at the interface but the normal traction and normal particle velocity are continuous, i.e., $T_{x z}^{\mathrm{I}}$ $=T_{x z}^{\mathrm{II}}=0, T_{y z}^{\mathrm{I}}=T_{y z}^{\mathrm{II}}=0, T_{z z}^{\mathrm{I}}=T_{z z}^{\mathrm{II}}, V_{z}^{\mathrm{I}}=V_{z}^{\mathrm{II}}$, where the superscripts I an II denote the lower and upper layers on each side of the interface, respectively. Buried solid lubricant layers of HOPG can be considered as an example of such interface. ${ }^{7}$ These boundary conditions can be described in terms of the surface impedance tensor as $V_{z}^{\mathrm{I}}=T_{z z}^{\mathrm{I}} G_{33}^{\mathrm{I}-1}=V_{z}^{\mathrm{II}}$, where $G^{\mathrm{I}}$ is the surface impedance tensor at the top of the lower layer. Therefore, the only nonzero term in the surface impedance tensor $\mathrm{G}^{\mathrm{II}}$ at the bottom of layer II is given as

$$
G_{33}^{\mathrm{II}}=1 / G_{33}^{\mathrm{I}-1} \text {. }
$$

Using initial conditions in Eqs. (4) and (5) at the defective interfaces the surface impedance at the sample surface is calculated and the contact stiffness for the defective sample is evaluated. In the following calculations, a silicon AFM tip with $100 \mathrm{~nm}$ radius of curvature vibrating at $100 \mathrm{kHz}$ is assumed. For $\mathrm{SiO}_{2}$ film and $100 \mathrm{~nm}$ copper layer on silicon substrate, the contact force is $300 \mathrm{nN}$ and for other calcula-

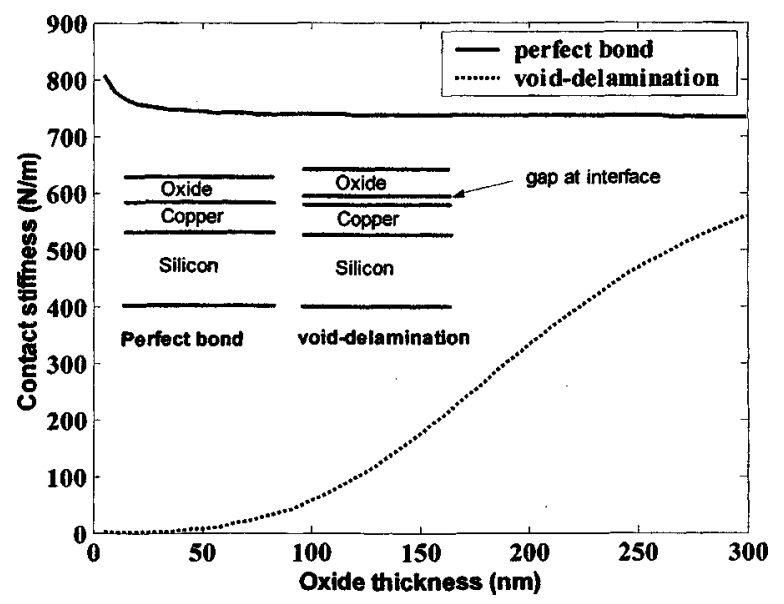

FIG. 1. Calculated contact stiffness as a function of $\mathrm{SiO}_{2}$ thickness in a multilayer electrical interconnect stack made of $\mathrm{SiO}_{2}$ dielectric layer and 100-nm-thick copper conductor on silicon substrate.

tions the contact force is $200 \mathrm{nN}$. Figure 1 shows the calculated contact stiffness variation as a function of $\mathrm{SiO}_{2}$ thickness in a multilayer electrical interconnect stack made of $\mathrm{SiO}_{2}$ dielectric layer and 100-nm-thick copper conductor on silicon substrate. When the layers are perfectly bonded, the contact stiffness is reduced and the contact radius is increased with increasing $\mathrm{SiO}_{2}$ layer thickness as the more compliant $\mathrm{SiO}_{2}$ film starts to dominate the surface stiffness. This would result in a downshifting of cantilever resonance frequency in an ultrasonic AFM experiment as reported earlier. ${ }^{12}$ As also shown in Fig. 1, when there is a delamination or void defect at the $\mathrm{SiO}_{2} / \mathrm{Cu}$ interface, the AFM essentially measures the stiffness of the top $\mathrm{SiO}_{2}$ layer. Since experiments show that changes in contact stiffness in the $0.5-1 \%$ range can be readily resolved by ultrasonic AFM, the results indicate a void defect buried nearly 500-nm-deep dielectric layer can be detected. ${ }^{7,12}$ It has to be noted that this figure is valid for defects with a lateral size much larger than the contact radius, which can be the case for electromigration induced voids. $^{10}$

Figure 2 shows the variation of contact stiffness with perfect bond and disbond conditions at the interface between tungsten or aluminum films and silicon substrate. In case of a perfect bond, the contact stiffness increases for the tungsten and decreases for aluminum films, respectively, as expected. When a disbond defect is introduced at the thin film-silicon interface using the initial condition in Eq. (5), the model

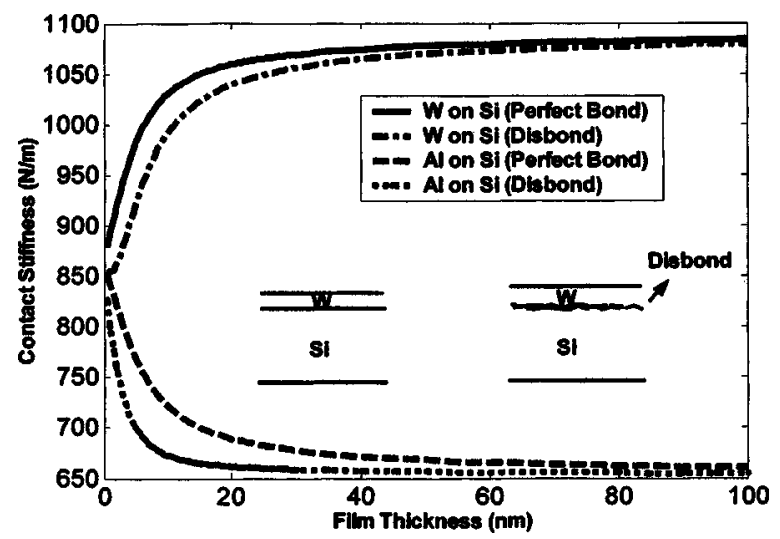

FIG. 2. Calculated normal contact stiffness for perfect bond and disbond for aluminum layer-silicon substrate and tungsten layer-silicon substrate. 


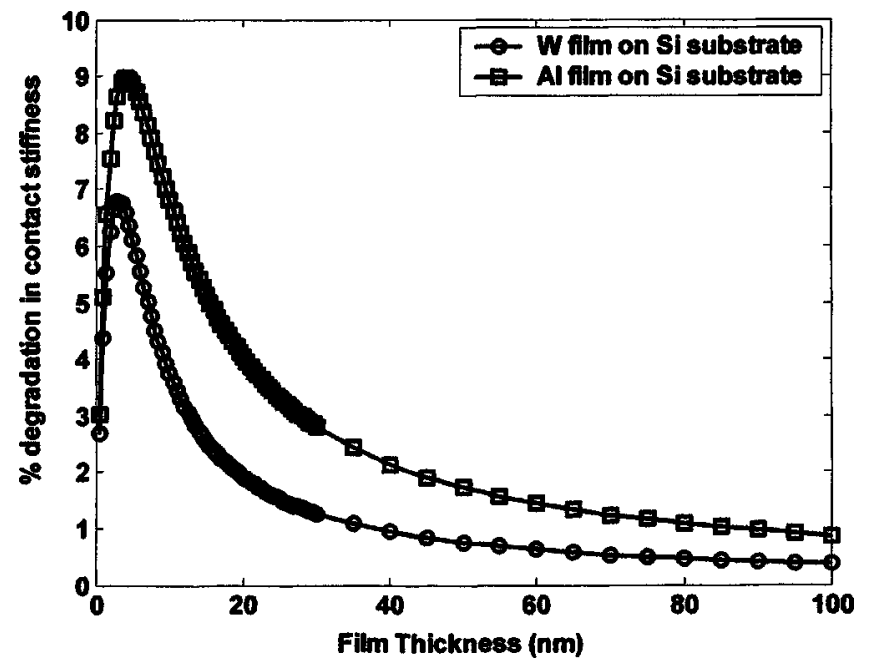

FIG. 3. Percentile change in normal contact stiffness for disbond between tungsten layer-silicon half space and aluminum layer-silicon half space as a function of defect depth.

predicts that the contact stiffness is further reduced as compared to the perfect bond case for both films. This is also in agreement with experimental results obtained on $\mathrm{HOPG}^{7} \mathrm{An}$ optimum defect depth is calculated by normalizing the contact stiffness for a sample with defect to that of without defect and plotting this percentile change as a function of film thickness. Figure 3 shows these results for tungsten and aluminum films on silicon half space. Although the optimum defect depth is on the order of a few nanometers and the ultrasonic AFM is quite sensitive for disbond defects buried less than $20 \mathrm{~nm}$ below surface, defects as deep as $100 \mathrm{~nm}$ should be detectable.

The detectable defect depth could be extended by increasing the applied force on the surface or the radius of curvature of the tip. These parameters appear in the form of a product in Hertzian contact theory and Fig. 4 shows that increasing the product increases the contact radius and the range of the evanescent waves generated by the AFM tip enabling deeper defects to be sensed. Interestingly, the maximum amount of percentile change in contact stiffness between the AFM tip and the surface seems independent of the applied force and the radius of curvature of the tip. Obvi-

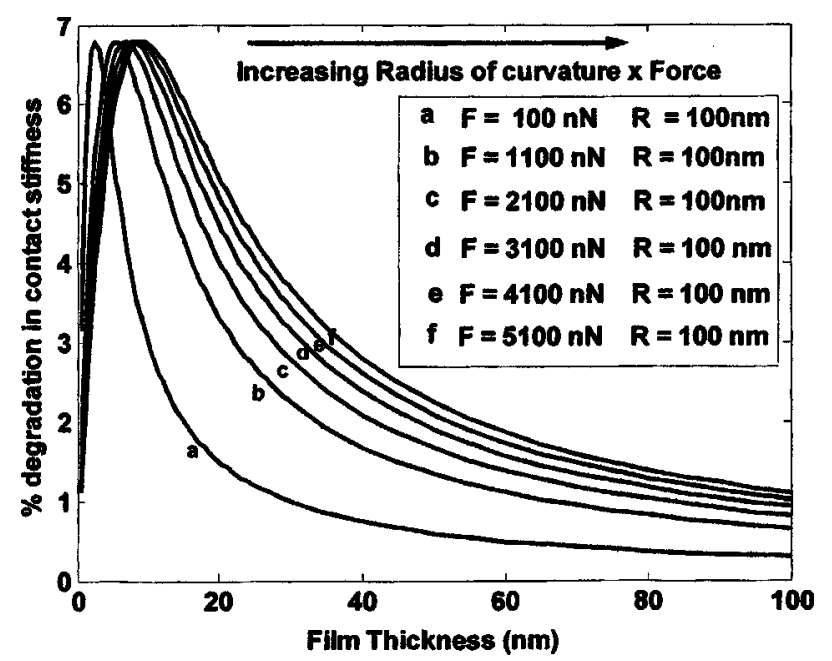

FIG. 4. Percentile change in normal contact stiffness for disbond between tungsten film and silicon half space as a function of defect depth for various force-radius of curvature products.

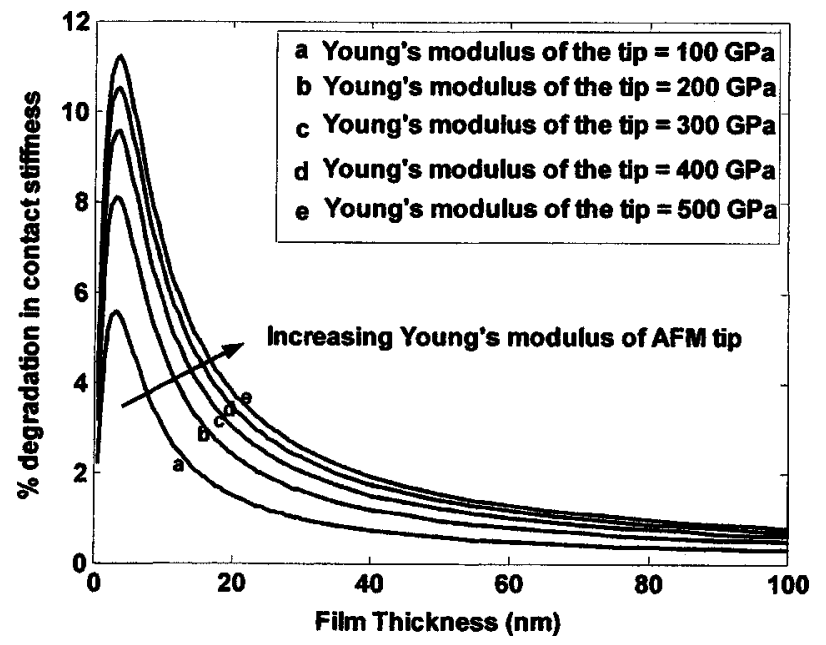

FIG. 5. Percentile change in normal contact stiffness for disbond between tungsten film and silicon half space as a function of defect depth for various tip materials.

ously, one needs to limit the amount of contact force so as not to damage the sample. The effect of the AFM tip material on defect detection for tungsten film-silicon substrate structure is also investigated and the results are presented in Fig. 5.

In summary, we have provided an analytical formulation for the sensitivity of AFM to several types of subsurface interface defects. The results show that ultrasonic AFM techniques measuring normal contact stiffness should resolve delaminations and voids typically found at the various interfaces of electrical interconnects. Although it can be improved at the cost of lateral resolution, the detection depth of ultrasonic AFM is lower for disbonds at subsurface interfaces. We are currently working on improving the penetration depth for disbonded interfaces using an ultrasonic AFM to measure lateral contact stiffness, since lateral contact stiffness is expected to be more sensitive to disturbances in shear stress discontinuities at subsurface interfaces.

The authors would like to thank G.G. Yaralioglu of Ginzton Laboratory, Stanford University for his helpful comments.

${ }^{1}$ N. A. Burnham, A. J. Kulik, G. Gremaud, P. J. Gallo, and F. Oulevey, J. Vac. Sci. Technol. B 14, 794 (1996).

${ }^{2}$ O. Sahin and A. Atalar, Appl. Phys. Lett. 79, 4455 (2001).

${ }^{3}$ K. Yamanaka, H. Ogiso, and O. Kolosov, Appl. Phys. Lett. 64, 178 (1994).

${ }^{4}$ M. Kopycinska, C. Ziebert, H. Schmitt, U. Rabe, S. Hirsekorn, and W. Arnold, Surf. Sci. 532, 450 (2003).

${ }_{5}^{5}$ A. G. Onaran, F. L. Degertekin, and B. Hadimioglu, Appl. Phys. Lett. 80, 4063 (2002).

${ }^{6}$ G. G. Yaralioglu, F. L. Degertekin, K. B. Crozier, and C. F. Quate, J. Appl. Phys. 87, 7491 (2000).

${ }^{7}$ T. Tsuji and K. Yamanaka, Nanotechnology 12, 301 (2001).

${ }^{8}$ A. P. McGuigan, B. D. Huey, G. A.D. Briggs, O. V. Kolosov, Y. Tsukahara, and M. Yanaka, Appl. Phys. Lett. 80, 1180 (2000).

${ }^{9}$ L. Muthuswami, E. S. Moyer, Z. Li, and R. E. Geer, Proceedings of the IEEE Interconnect Technology Conference, 2002, p. 239.

${ }^{10}$ G. Xu, X. Su, C. B. Stagarescu, D. E. Eastman, B. Lai, Z. Cai, I. C. Noyan, and C. K. Hu, Appl. Phys. Lett. 78, 820 (2001).

${ }^{11}$ T. Scherban, B. Sun, J. Blaine, C. Block, B. Jin, and E. Andideh, Proceedings of the IEEE Interconnect Technology Conference, 2001, p. 257.

${ }^{12}$ K. B. Crozier, G. G. Yaralioglu, F. L. Degertekin, J. D. Adams, S. C. Minne, and C. F. Quate, Appl. Phys. Lett. 76, 1950 (2000).

${ }^{13}$ F. L. Degertekin, Ph.D. thesis, Stanford University, 1997, also see F. L. Degertekin, J. Pei, B. V. Honein, B. T. Khuri-Yakub, and K. C. Saraswat, Proceedings of the IEEE Ultrasonics Symposium, 1994, p. 1337. 